\title{
A second-order impact model for forest fire regimes
}

Stefano Maggi (maggi@@elet.polimi.it)

Sergio Rinaldi (sergio.rinaldi@polimi.it)

\section{Approved by}

Ulf Dieckmann

Program Leader, Evolution and Ecology Program

December 2006 


\section{Contents}

Abstract. 1 Introduction 


\title{
A second-order impact model
}

\section{for forest fire regimes}

\author{
Stefano Maggi ${ }^{\text {a }}$, Sergio Rinaldi ${ }^{\mathrm{a}, \mathrm{b}, *}$ \\ ${ }^{a}$ Dipartimento di Elettronica e Informazione, Politecnico di Milano, Milano, Italy. \\ ${ }^{b}$ Evolution and Ecolgy Program, International Institute for Applied Systems
}

Analysis, 2361 Laxenburg, Austria.

\begin{abstract}
We present a very simple "impact" model for the description of forest fires and show that it can mimic the known characteristics of wild fire regimes in savannas, boreal forests, and Mediterranean forests. Moreover, the distribution of burned biomasses in model generated fires resemble those of burned areas in numerous large forests around the world. The model has also the merits of being the first second-order model for forest fires and the first example of the use of impact models in the study of ecosystems.
\end{abstract}

Keywords: forest fires, savannas, boreal forests, Mediterranean forests, impact models, chaos

Corresponding author. Address: Dipartimento di Elettronica e Informazione, Politecnico di Milano, Via Ponzio 34/5, 20133, Milano, Italy. Phone: +39 0223993563 , Fax: +3902 23993412.

Email addresses: maggi@elet.polimi.it (Stefano Maggi), rinaldi@elet.polimi.it (Sergio Rinaldi). 


\section{Introduction}

In savannas, as well as in boreal and in Mediterranean forests, wild fires are recurrent, but with remarkably different characteristics. Fires in savannas are almost periodic surface fires with return times ranging from 1-2 yr in moist areas (Goldammer (1983)) to 5-10 yr in arid areas (Rutherford (1981)). Fires in northern boreal forests are also quite regular, but they prevalently involve crowns (Kasischke et al. (1995)) and occur every 50-200yr (Rowe and Scotter (1973); Zackrisson (1977); Engelmark (1984); Payette (1989)). By contrast, in Mediterranean areas, mixed (crown and surface) fires are almost the rule and occur in an apparently random fashion, with highly variable return times (Kruger (1983); Davis and Burrows (1994)).

While it is true that natural forest fires originate from random events (mostly lightning) and are influenced by meteorological conditions (Bessie and Johnson (1995)), it is also true that fires can develop only if there is enough dry matter on the ground and if plants are sufficiently abundant in at least one of the various vegetational layers of the forest (for a relatively detailed discussion of this issue see Casagrandi and Rinaldi (1999) and references therein). This suggests the idea that long-term predictions of forest fires can be roughly performed with deterministic models describing the growth processes, while more precise short-term predictions can only be performed through stochastic models (conceptually comparable with those used in weather forecast).

Here we propose a simple deterministic model for the long-term prediction of forest fires in which the vegetational growth is described by standard ordinary differential equations, while fire episodes are modeled as instantaneous events. 
The fire develops when there is enough fuel on the ground, and, under suitable assumptions, this occurs when the mix of biomasses of the various layers reaches pre-specified values. The consequence of a fire is therefore an instantaneous reduction of the biomasses which is heuristically described by a simple rule in state space. Models with discontinuities of this kind are called "impact models" and have been first used in mechanics (see Brogliato (1999) and references therein) to describe the dynamics of mechanical systems characterized by impacts among various masses. They are quite special and can be used to explain a number of rather subtle phenomena like the "Zeno chattering" (e.g., the diminishing return times of the impacts of a ping-pong bouncing ball) that other models can not explain. Impact models represent the most naïve approach for the description of systems characterized by dynamic phenomena occurring at very diversified time scales (in our case the slow building up of biomass and its fast destruction through fire). They should not be confused with an apparently similar but substantially different class of models, namely that of periodically pulsed systems where the discontinuity in state space is generated by a periodic exogenous shock on the system. Many are the examples of this second class of models in biology: the control of continuously stirredtank reactors (Funasaki and Kot (1993)), the study of pulsed chemotherapy (Lakmeche and Arino (2001)) and vaccination (Shulgin et al. (1998)) and a number of contributions dealing with the effects of periodic harvesting or immigration (Ives et al. (2000); Liu and Chen (2003); Chau (2000); Grant et al. (1997); Geritz and Kisdi (2004); Reluga (2004)). However, in those models the return time of the discontinuous event is constant and a-priori fixed, while in our impact model the fire return times are neither constant nor pre-specified but are endogenously created by the interactions among the various layers of the forest. 
The impact model we propose in this paper has only two differential equations (i.e. it is a so-called second-order model), one for the lower and one for the upper layer of the forest. We therefore exclude from our study singlelayered shrublands. The simplest deterministic model available until now for the study of the dynamics of the fire return times was a standard (i.e. non impact) fourth-order model (Casagrandi and Rinaldi (1999)) in which the four state variables are the burning and non burning biomasses of the lower and upper layers of the forest. It is important to keep in mind that the impact model we propose in this paper should not be intended as an approximation to that fourth-order model. However, it can mimic the qualitative features of the periodic fire regimes of savannas and boreal forest, as well as the chaotic fire regimes of Mediterranean forests suggested by the fourth-order model. As far as we know, this is also the first time that the general idea behind impact models is applied in ecology, although forestry and agricultural practices correspond very closely to the same idea: harvest when the population reaches a specified state. For this reason, it would be surprising if related models have not been applied in that context. In any case, there are certainly many other potential applications, since population dynamics are very often the result of slow dynamical processes interrupted by short devastating events.

\section{The model}

A continuous-time impact model is described by a set of $n$ ordinary differential equations

$$
\dot{x}(t)=f(x(t))
$$


which hold at any point in state space except on a $(n-1)$ dimensional manifold $X^{-}$, where the impact occurs. When the state $x$ reaches the manifold $X^{-}$at point $x^{-}$, an instantaneous transition described by a map

$$
x^{+}=\varphi\left(x^{-}\right) \quad x^{-} \in X^{-}
$$

occurs. The set

$$
X^{+}=\varphi\left(X^{-}\right)
$$

is the set of the states of the system immediately after the impact. For this reason, the sets $\mathcal{X}^{-}$and $\mathcal{X}^{+}$are called, in general, pre- and post-impact manifolds. In the specific application considered in this paper, they simply represent the pre- and post-fire conditions of the forest and are therefore called pre- and post-fire manifolds. Obviously, first-order impact models are of no interest because if $n=1$ the manifolds $X^{-}$and $X^{+}$are just two points and their most complex behavior is just a cycle passing through $X^{-}$and $X^{+}$. This is why impact models are usually presented for $n \geq 2$.

The model we propose is a crude simplification of the real world. Species diversity, age structure, spatial heterogeneity, and plant physiology are not taken into account since we look only at total biomasses (see Shugart (1984), chap. 6 for a discussion). However, in order to distinguish fires in different layers, we assume that the forest is composed of two layers: a lower vegetational layer (from now on called "bush") that, depending on the forest, is composed of bryophytes, herbs, shrubs, or any mix of these plants, and an upper vegetational layer (from now on called "tree"), in general composed of plants of various species. The corresponding biomasses are denoted by $B$ (bush) and $T$ 
(tree). The equations of growth (1) characterizing our model are

$$
\begin{aligned}
& \dot{B}=r_{B} B\left(1-\frac{B}{K_{B}}\right)-\alpha B T \\
& \dot{T}=r_{T} T\left(1-\frac{T}{K_{T}}\right)
\end{aligned}
$$

This means that, in the absence of fire, trees grow logistically toward the carrying capacity $K_{T}$, while plants of the lower layer do not tend toward their carrying capacity $K_{B}$ because tree canopy reduces light availability. A detailed discussion of the validity and limitations of eqs. (3) can be found in Casagrandi and Rinaldi (1999), where realistic values for the 5 vegetational parameters $\left(r_{B}, r_{T}, K_{B}, K_{T}, \alpha\right)$ are also suggested.

As for the fire, we know (see, for example, Viegas (1998)) that the ignition phase is possible if there is enough dead biomass on the ground (leaves, twigs, branches, moss, herbs, ...). Since the biochemical processes regulating the mineralization of dead biomass are relatively fast with respect to plant growth (Esser et al. (1982); Seastedt (1988)), we can reasonably assume, on the timescale at which we describe bush and tree growth, that the rate of mineralization (proportional to the amount of dead biomass) equals the inflow rate of new necromass into the ground layer (proportional to bush and tree biomass). As a result, we can consider $B$ and $T$ as appropriate indicators of the abundance of fuel on the ground. Of course, also the water content of the fuel particles matters, and, indeed, models for short-term fire prediction often include a number of relevant factors related with soil moisture. However such factors vary at high frequency and can therefore be neglected if the target is longterm fire prediction (in other words, if we like to predict within how many years (or decades) we are going to experience a new fire we can obviously forget high frequency phenomena like weekly weather variability). 
After this premise, we can specify (see Fig. 1(a)) the pre- and post-fire manifolds $X^{-}$and $X^{+}$and the map (2) interpreting the impact of the fire.

Fig. 1 about here

Let us first focus on the pre-fire conditions by noticing that the function $T(B)$ identifying the manifold $\mathcal{X}^{-}$is piecewise linear and non increasing and that the set below the manifold $\mathcal{X}^{-}$is convex. The first property is obvious because less fuel originated from trees (i.e. less trees) is necessary for fire ignition if more fuel originated from bushes is available on the ground.

The second property simply says that if $x^{\prime}=\left(B^{\prime}, T^{\prime}\right)$ and $x^{\prime \prime}=\left(B^{\prime \prime}, T^{\prime \prime}\right)$ are two states of the forest at which fire ignition is not possible (i.e. two points below the manifold $X^{-}$) no mix of these two states (i.e. no points of the segment connecting $x^{\prime}$ with $x^{\prime \prime}$ ) can give rise to fire ignition. A formal support to these two properties, which are here assumed to hold, is available in the Appendix.

Intuition suggests that $X^{-}$should be a smooth manifold while our choice (see Fig. 1) has been in favor of a less realistic but simpler piecewise linear manifold $X^{-}$. The reason for this choice is that our manifold $X^{-}$allows one to sharply identify surface fires (vertical segment of $\mathcal{X}^{-}$), crown fires (horizontal segment of $X^{-}$) and mixed fires (central segment of $X^{-}$). By definition, surface fires do not involve the upper layer, so that the post-fire conditions are on the vertical segment characterized by $B^{+}=\lambda_{B} \rho_{B} K_{B}=\lambda_{B} B^{-}$. In other words, $\rho_{B}$ is the proportion of the lower layer carrying capacity $K_{B}$ at which surface fires occur and $\lambda_{B}$ is the proportion of the lower layer biomass that survives to surface fires. Similarly, fires in the upper layer are characterized by a vertical instantaneous transition from $T^{-}=\rho_{T} K_{T}$ to $T^{+}=\lambda_{T} T^{-}$. The most extreme 
surface fire is represented by the transition $S^{-} \rightarrow S^{+}$, while the most extreme crown fire is represented by the transition $C^{-} \rightarrow C^{+}$. The assumption that mixed fires initiate on the segment $C^{-} S^{-}$implies, by continuity, that post-fire conditions are on a curve connecting points $C^{+}$and $S^{+}$. Of course, mixed fires initiating close to point $C^{-}$should end up close to point $C^{+}$. Since we have been unable to find simple suggestions on the shape of the manifold $X^{+}$ from point $C^{+}$to point $S^{+}$, we have assumed for simplicity that mixed-fires terminate on the segment $C^{+} S^{+}$and preserve the relative distances $\eta$ and $(1-\eta)$ from the two extreme points. In formulas, the extreme points of the pre-fire segment $C^{-} S^{-}$are

$$
C^{-}=\left(\sigma_{B} K_{B}, \rho_{T} K_{T}\right) \quad S^{-}=\left(\rho_{B} K_{B}, \sigma_{T} K_{T}\right)
$$

and each mixed fire starts from a point $\left(B^{-}, T^{-}\right)$belonging to the segment $C^{-} S^{-}$, i.e.

$$
\begin{aligned}
& B^{-}=\eta \sigma_{B} K_{B}+(1-\eta) \rho_{B} K_{B} \\
& T^{-}=\eta \rho_{T} K_{T}+(1-\eta) \sigma_{T} K_{T}
\end{aligned}
$$

where $0 \leq \eta \leq 1\left(\eta=0\right.$ and $\eta=1$ correspond to points $S^{-}$and $C^{-}$, respectively). The extreme points of the post-fire segment $C^{+} S^{+}$are

$$
C^{+}=\left(\sigma_{B} K_{B}, \lambda_{T} \rho_{T} K_{T}\right) \quad S^{+}=\left(\lambda_{B} \rho_{B} K_{B}, \sigma_{T} K_{T}\right)
$$

and the post-fire conditions are

$$
\begin{aligned}
& B^{+}=\eta \sigma_{B} K_{B}+(1-\eta) \lambda_{B} \rho_{B} K_{B} \\
& T^{+}=\eta \lambda_{T} \rho_{T} K_{T}+(1-\eta) \sigma_{T} K_{T}
\end{aligned}
$$

Thus, the map $x^{+}=\varphi\left(x^{-}\right)$for mixed fires is nothing but the transformation of point $\left(B^{-}, T^{-}\right)$into point $\left(B^{+}, T^{+}\right)$. This completes the description of the 
model. Notice that Fig. 1 has been drawn for the case $\sigma_{T}>\lambda_{T} \rho_{R}$ and $\sigma_{B}>$ $\lambda_{B} \rho_{B}$ but that eqs. $(4,5)$ hold for all possible cases.

The sequence of the fires can be easily obtained from the model, as shown in Fig. 1(b). Starting from a given initial condition, say point 0 in Fig. 1(b), one integrates the differential equations (3) until the solution hits the pre-fire manifold $X^{-}$at point $\left(B^{-}, T^{-}\right)$(see point $1^{-}$in Fig. $\left.1(\mathrm{~b})\right)$. From anyone of the two equations (4) one can derive the value of $\eta$ associated with point $1^{-}$ and then use eqs. (5) for computing point $1^{+}$. Then, the procedure is iterated and a series of fires $2^{-} \rightarrow 2^{+}, 3^{-} \rightarrow 3^{+}, \ldots$ is obtained.

As pointed out in Fig. 1(b), the trajectory of the system is the concatenation of slow transitions (continuous lines) corresponding to growing phases, and fast (actually instantaneous) transitions (dotted lines) corresponding to fires. It is worth noticing that fast transitions can intersect with slow and fast transitions. This is why the model can be chaotic even if it is only a second-order model.

\section{Results}

In this section we show that our second-order model can mimic, for suitable values of its parameters, the characteristic fire regimes of savannas, boreal forests, and Mediterranean forests. For each kind of forest we present the result of a typical simulation and compare it with the result obtained with the more complex fourth-order model (Casagrandi and Rinaldi (1999)). Simulations were performed with 4th order Runge-Kutta-Fehlberg method with

5th order error estimate. Impacts of the trajectory with the pre-fire manifold $X^{-}$were detected with an adaptive step integration procedure with absolute 
tolerance of $10^{-9}$. Then, we also show that the distributions of the burned biomasses in model generated fires have structural properties quite similar to those emerging from statistical analysis of the areas burned by fires in numerous large forests around the world.

\section{Savannas}

A typical series of fires in savannas obtained through simulation of the secondorder model is shown in Fig. 2(a). The fires occur every 8 yrs, in good agreement with Rutherford (1981). The cycle, shown in Fig. 2(b), is set up by the lower layer, and in fact the fire is essentially a surface fire devastating the herbs (the post-fire bush biomass is only $10 \%$ of the pre-fire bush biomass), while tree biomass remains almost constant, as observed by Hopkins (1965).

The second row of Fig. 2 shows very similar results obtained with the fourthorder model.

Fig. 2 about here

\section{Boreal forests}

A typical fire regime of a boreal forest obtained with our impact model is shown in Fig. 3(a)-(b). In agreement with many data and studies on boreal forests at high latitudes, the fires are essentially crown fires and occur every 100 yrs (Yarie (1981)). After a fire, the biomass of the lower layer increases for 20-30 yrs (while conifers grow very slowly) and then decreases and reaches the pre-fire level (manifold $\mathcal{X}^{-}$in Fig. 3(b)) within 60-100 yrs as predicted by 
Viereck (1983).

A very similar fire regime obtained with the fourth-order model is shown in Fig. 3(c)-(d). Obviously, the two models do not explain special surface fires (observed in boreal forests), due to litter accumulation on the ground (Kilgore and Taylor (1979)).

Fig. 3 about here

\section{Mediterranean forests}

Using suitable parameter values our impact model points out chaotic fire regimes. The first row of Fig. 4 shows the strange attractor (b) and a 200 yrs long time series (a) extracted from the strange attractor. Fires are recurrent but not periodic: the fire return times vary from 10 to 50 yrs, in good agreement with a number of studies on two layers forests (Hanes (1971); Le Houérou (1974); Keeley (1977); Schlesinger and Gill (1978); Horne (1981)). Moreover, some of the fires are surface fires (indicated by S in Fig. 4(a)), while others are mixed fires (indicated by $\mathrm{M}$ in Fig. 4(a)). Again, quite similar results can be obtained with the fourth-order model (see second row of Fig. $4)$.

Fig. 4 about here

Comparison with field data

The comparison of the fire regimes proposed by our second-order model with real fire regimes is very difficult, if not impossible. A first difficulty is that the 
model describes the behavior of a natural forest, while the great majority of available data refer to forests where fire-fighting efforts were systematically performed. A second and more important obstacle is that data do not refer to burned biomasses but rather to burned areas which are largely influenced by meteorological conditions (Bessie and Johnson (1995)). Under these circumstances, we can at most hope that some relevant features of forest fires (detectable from statistical analyses of field data) have some sort of analogy in the model behavior. The most relevant of such features is, undoubtedly, the power-law distribution of the burned areas. The power law has been first suggested on the basis of a rather naive interpretation of forest fires in terms of probabilistic cellular automata (Bak et al. (1990); Drossel and Schwabl (1992)) and then supported by some statistical analyses of field data (Malamud et al. (1998); Ricotta et al. (1999)). This law would imply that the log-log plot of the cumulative distribution of the burned areas is a straight line. However, more recently, Ricotta et al. (2001), Reed and McKelvey (2002) and Telesca et al. (2005) have shown through statistical analysis of extensive fire records concerning Mediterranean areas in Italy, Spain, Corsica and Greece and six regions in North America (Sierra Nevada, Nez Perce, Clearwater, Yosemite, N. E. Alberta, North West Territories) that the log-log plot of the cumulative distribution is not a straight line but can be approximated by three straight segments, as shown in the first row of Fig. 5. It is therefore very interesting to note (see Fig. 5(c)-(d)) that quite similar log-log plots can be obtained by computing the cumulative distributions of burned biomasses in model generated fires.

Fig. 5 about here 
Moreover, a detailed analysis of the fires generated by the model shows that the fires associated to flat segments of the cumulative distributions are mainly surface fires while those associated to the steepest part of the cumulative distributions are crown fires. This suggests that the property empirically pointed out by Ricotta et al. (2001), Reed and McKelvey (2002) and Telesca et al. (2005) could simply be related with forest morphology, i.e. with the existence of various layers.

\section{Discussion}

We have presented a second-order model capable of mimicking all the main forest fire regimes. This is a quite appreciable result since many problems in ecology have, since long, their paradigmatic second-order model (e.g. LotkaVolterra models for competition and predation, Leslie model for two stage populations, Streeter-Phelps model for biodegradable pollution in rivers and lakes, SIR model for epidemics, ... ), forest fires still did not have it.

To our knowledge, the model is also the first impact model proposed in ecology.

Other impact models could possibly be used for other ecosystems in which fast and devastating events recursively occur. Promising applications are plankton blooms in shallow lakes and insect-pest outbreaks in forests.

Our analysis has shown that the proposed model is rather flexible and can be adapted to savannas, boreal forests and Mediterranean forests. Moreover, the statistical characteristics of the intensity of the fires generated by the model resemble those emerging from the statistical analysis of large data sets of various forests in Mediterranean countries and in North America. 
The analysis of the bifurcations of the model is of great interest, since it would produce in a systematic way the whole catalogue of fire regimes described by the model. However, the solution of this problem is far from being trivial, since the theory of bifurcations of impact models is still incomplete and, on the top of this, our manifolds $X^{-}$and $X^{+}$are not smooth. A first attempt in this direction (Dercole and Maggi (2004)) has pointed out that the model is sensitive to the parameters $\lambda_{B}$ and $\lambda_{T}$ which are the proportions of surface and tree biomass that survive to surface and crown fires, respectively. In particular, the analysis has shown that the transition from chaotic fire regimes (typical of Mediterranean forests) to cyclic fire regimes (typical of savannas and boreal forests) can be rather sharp and interpretable as a so-called border collision bifurcation.

Some problems concerning the model could be further explored. The analysis of one or more stochastic versions of the model would be interesting, for example by letting the set $\mathcal{X}^{-}$of pre-fire conditions depend upon meteorological conditions. This would certainly amplify the chaoticity of the deterministic component of the system, thus giving to weather variability the role it deserves (Bessie and Johnson (1995)). The model could also be used to determine, at least qualitatively, the most important effects that climate change and different management policies (e.g. thinning, grazing, remote monitoring, etc.) have on fire frequencies and intensities. Another issue of practical interest, that could be explored with our impact model, is the description of fire propagation in spatially extended forests. 


\section{Acknowledgments}

The authors are grateful to Renato Casagrandi for his helpful suggestions and

for his criticisms on a first draft of the paper and to one of the anonymous reviewers who allowed us to improve the quality of the paper. Financial support was provided by MIUR under project FIRB2001-RBNE01CW3M. 


\section{References}

Bak, P., Chen, K., Tang, C., 1990. A forest-fire model and some thoughts on turbulence. Physics Letters A 147, 297-300.

Bessie, W. C., Johnson, E. A., 1995. The relative importance of fuels and weather on fire behavior on subalpine forests. Ecology 76, 747-762.

Brogliato, B., 1999. Nonsmooth Mechanics, 2nd Edition. Springer-Verlag.

Casagrandi, R., Rinaldi, S., 1999. A minimal model for forest fire regimes. Am. Nat. 153, 527-539.

Chau, N. P., 2000. Destabilising effect if periodic harvest on population dynamics. Ecological Modelling 127, 1-9.

Davis, F. W., Burrows, D. A., 1994. Spatial simulation of fire regime in Mediterranean-climate landscapes. In: Moreno, J. M., Oechel, W. C. (Eds.), The role of fire in Mediterranean type ecosystems. Springer, New York, pp. 117-139.

Dercole, F., Maggi, S., 2004. Detection and continuation of critical bifurcations in impact models. Applied Mathematics and Computation 168 (1), 623-635.

Drossel, B., Schwabl, F., 1992. Self-organized critical forest-fire model. Physical Review Letters 69, 1629-1632.

Engelmark, O., 1984. Forest fires in the Muddus National Park (Northern Sweden) during the past 600 years. Canadian Journal of Botany 62, 893898.

Esser, G., Aselmann, I., Lieth, H., 1982. Modelling the carbon reservoir in the system compartment 'litter'. Mitteilungen aus dem GeologischPaleontologishen Institut der Universitaet Hamburg 52, 39-58.

Funasaki, E., Kot, M., 1993. Invasion and chaos in a periodically pulsed massaction chemostat. Theoretical Population Biology 44, 203-224. 
Geritz, S. A. H., Kisdi, E., 2004. On the mechanistic underpinning of discretetime population models with complex dynamics. Journal of Theoretical Biology 228, 261-269.

Goldammer, J. G. (Ed.), 1983. Fire in tropical biota: Ecosystems processes and global challenges. Springer, Berlin.

Grant, W. E., Pedersen, E. K., Marin, S. L., 1997. Ecology and Natural Resource Management: System Analysis and Simulation. Wiley, New York.

Hanes, T. L., 1971. Succession after fire in the chaparral of southern California. Ecological Monographs 41, 27-52.

Hopkins, B., 1965. Observations on savanna burning in the Olokemeji Forest Reserve, Nigeria. Journal of Applied Ecology 2, 367-381.

Horne, I. P., 1981. The frequency of veld fires in the Groot Swartberg mountain catchment area, Cape Province. South African Forestry Journal 118, 56-60.

Ives, A. R., Gross, K., Jansen, V. A. A., 2000. Periodic mortality events in predator-prey systems. Ecology 81, 3330-3340.

Kasischke, E. S., Christiensen, N. L., Stocks, B. J., 1995. Fire, global warming, and the carbon balance of boreal forests. Ecological applications 5, 437-451.

Keeley, J. E., 1977. Seed production, seed populations in soil and seedling production after fire for two generic pairs of sprouting and nonsprouting chaparral shrubs. Ecology 58, 820-829.

Kilgore, B. M., Taylor, D., 1979. Fire history of a mixed conifer forest. Ecology $60,129-142$.

Kruger, F. J., 1983. Plant community diversity and dynamics in relation to fire. In: Kruger, F. J., .Mitchell, D. T., Jarvis, J. U. (Eds.), Mediterraneantype ecosystems. Springer, Berlin, pp. 446-473.

Lakmeche, A., Arino, O., 2001. Nonlinear mathematical model of pulsedtherapy of heterogeneous tumors. Nonlinear Analysis Real World Appl. 2, 
455-465.

Le Houérou, M., 1974. Fire and vegetation in the Mediterranean Basin. In: Tall Timbers Fire Ecology Conference Proceedings. Vol. 13. pp. 237-277.

Liu, X., Chen, L., 2003. Complex dynamics of Holling type II Lotka-Volterra predator-prey system with impulsive perturbations on the predator. Chaos, Solitons and Fractals 16 (2), 311-320.

Malamud, B. D., Morein, G., Turcotte, D. L., 1998. Forest fires: An example of self-organized critical behavior. Science 281, 1840-1842.

Payette, S., 1989. Fire as a controlling process in the North American boreal forest. In: Shugart, H. H., Leemans, R., Bonan, G. B. (Eds.), A systems analysis of the global boreal forest. Cambridge University Press, Cambridge, pp. 145-169.

Reed, W. J., McKelvey, K. S., 2002. Power-law behavior and parametric models for the size-distribution of forest fires. Ecological Modelling 150, 239-254.

Reluga, T. C., 2004. Analysis of periodic growth-disturbance models. Theoretical Population Biology 66, 151-161.

Ricotta, C., Arianoutsou, M., Díaz-Delgado, R., Dugut, B., Lloret, F., Maroudi, E., Mazzoleni, S., Moreno, J. M., Rambal, S., Vallejo, R., Vázquez, A., 2001. Self-organized criticality of wildfires ecologically revisited. Ecological Modelling 141, 307-311.

Ricotta, C., Avena, G., Marchetti, M., 1999. The flaming sandpile: Selforganized criticality and wildfires. Ecological Modelling 119, 73-77.

Rowe, J. S., Scotter, G. W., 1973. Fire in the boreal forest. Quaternary research $8,444-464$.

Rutherford, M. C., 1981. Survival, regeneration and leaf biomass changes in woody plants following spring burns in Burkea africana-Ocnha pulchra savanna. Bothalia 13, 531-552. 
Schlesinger, W. H., Gill, D. S., 1978. Demographic studies of the chaparral shrubs, Ceanothus megacarpus, in the Santa Ynez Mountains. Ecology 59, $1256-1263$.

Seastedt, T., 1988. Mass, nitrogen, and phosphorous dynamics in foliage and root detritus of tallgrass prairie. Ecology 69, 59-65.

Shugart, H. H., 1984. A theory of forest dynamics. Springer, New York.

Shulgin, B., Stone, L., Agur, Z., 1998. Pulse vaccination strategy in the SIR epidemic model. Bulletin of Math. Biol. 60, 1-26.

Telesca, L., Amatulli, G., Lasaponara, R., Lovallo, M., Santulli, A., 2005. Time-scaling properties in forest-fire sequences observed in Gargano area (Southern Italy). Ecological Modelling 185, 531-544.

Viegas, D. X., 1998. Forest fire propagation. Phil. Trans. R. Soc. Lond. A 356, $2907-2928$.

Viereck, L. A., 1983. The effects of fire in black spruce ecosystems of Alaska and northern Canada. In: Wein, R. W., MacLean, D. A. (Eds.), The role of fire in northern circumpolar ecosystems. Wiley, Chichester, pp. 201-220.

Yarie, J., 1981. Forest fire cycle and life tables: a case of study from interior Alaska. Canadian Journal of Forest Research 11, 554-562.

Zackrisson, O., 1977. Influence of forest fires on the North Swedish boreal forest. Oikos 29, 22-32. 


\section{Figure captions}

Fig. 1 Model behavior. (a) The pre- and post-fire manifolds $X^{-}$and $X^{+}$; the dotted lines with double arrows are the instantaneous transitions from $\mathcal{X}^{-}$ to $\mathcal{X}^{+}$due to a fire (see eq. (2)); horizontal (vertical) lines correspond to surface (crown) fires in which trees (bushes) are not involved; oblique lines starting from the segment $C^{-} S^{-}$of $X^{-}$correspond to mixed fires. (b) State portrait of the model; continuous lines with a single arrow represent the growing phase of the forest and are described by eq. (3).

Fig. 2 Examples of fire regimes in savannas. First row: time series (a) and state portrait (b) obtained with the second-order model (parameter values: $r_{B}=1.5, r_{T}=1, K_{B}=0.4, K_{T}=0.5, \alpha=1.5, \rho_{B}=0.49, \rho_{T}=4$, $\left.\sigma_{B}=0.1, \sigma_{T}=0.985, \lambda_{B}=0.1, \lambda_{T}=0.2\right)$. Second row: time series (c) and state portrait (d) obtained with the fourth-order model (see Casagrandi and Rinaldi (1999) for parameter values).

Fig. 3 Examples of fire regimes in boreal forests. First row: time series (a) and state portrait (b) obtained with the second-order model (parameter values: $r_{B}=0.3, r_{T}=0.067, K_{B}=0.1, K_{T}=3, \alpha=0.045, \rho_{B}=10, \rho_{T}=0.98$, $\sigma_{B}=0.2, \sigma_{T}=0.1, \lambda_{B}=\lambda_{T}=0.01$ ). Second row: time series (c) and state portrait (d) obtained with the fourth-order model (see Casagrandi and Rinaldi (1999) for parameter values).

Fig. 4 Examples of fire regimes in Mediterranean forests. First row: time series (a) and state portrait (b) obtained with the second-order model (parameter values: $r_{B}=3 / 8, r_{T}=1 / 16, K_{B}=K_{T}=1, \alpha=129 / 800, \rho_{B}=0.85$, $\left.\rho_{T}=14 / 15, \sigma_{B}=0.6, \sigma_{T}=0.35, \lambda_{B}=\lambda_{T}=10^{-4}\right)$. Second row: time series (c) and state portrait (d) obtained with the fourth-order model (see Casagrandi and Rinaldi (1999) for parameter values). The arrows M and S 
point out mixed and surface fires.

Fig. 5 Examples of fire statistics. First row: cumulative distributions (number of fires) of burned areas in (a) Alicante region, 1973-1996 (obtained from Ricotta et al. (2001)) and (b) Gargano region, 1997-2003 (b) (obtained from Telesca et al. (2005)). Second row: cumulative distributions (proportion of fires) of burned biomasses obtained with the second-order model for two parameter settings $\left((\mathrm{c}): r_{B}=0.3, r_{T}=0.067, K_{B}=0.7, K_{T}=3, \alpha=0.03\right.$, $\rho_{B}=0.8605, \rho_{T}=0.98, \sigma_{B}=0.465, \sigma_{T}=0.4, \lambda_{B}=\lambda_{T}=10^{-4} ;(\mathrm{d}):$ as in (c) except $\left.K_{B}=1, \alpha=0.0375, \rho_{B}=0.84, \sigma_{T}=0.405\right)$. 

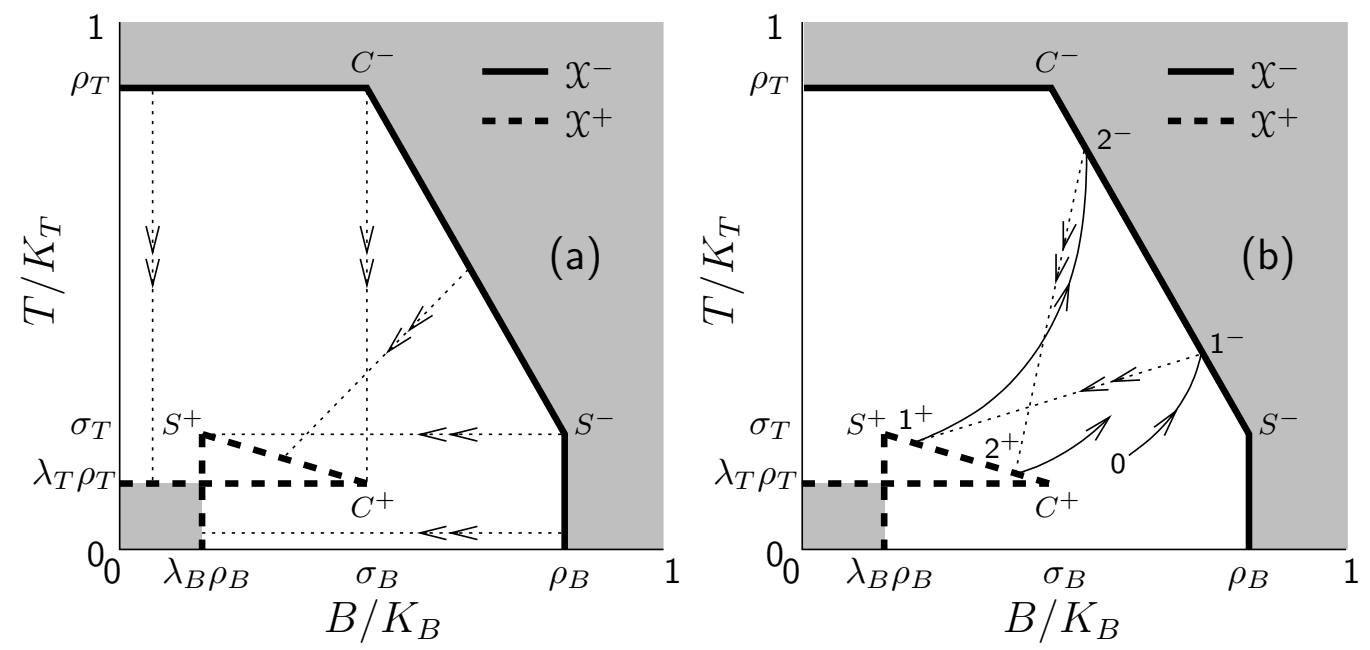

Figure 1. Maggi \& Rinaldi 2005 

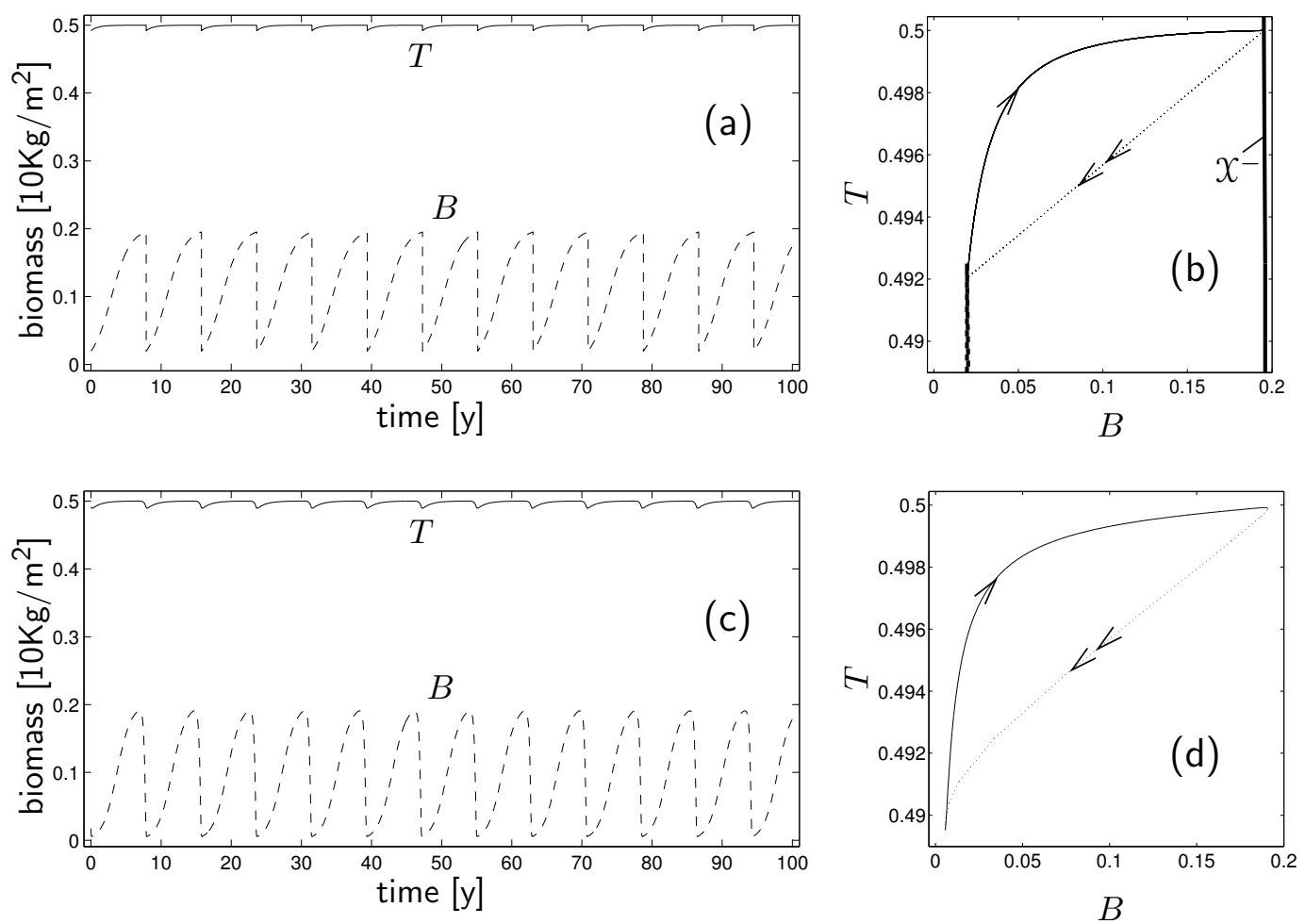

Figure 2. Maggi \& Rinaldi 2005 

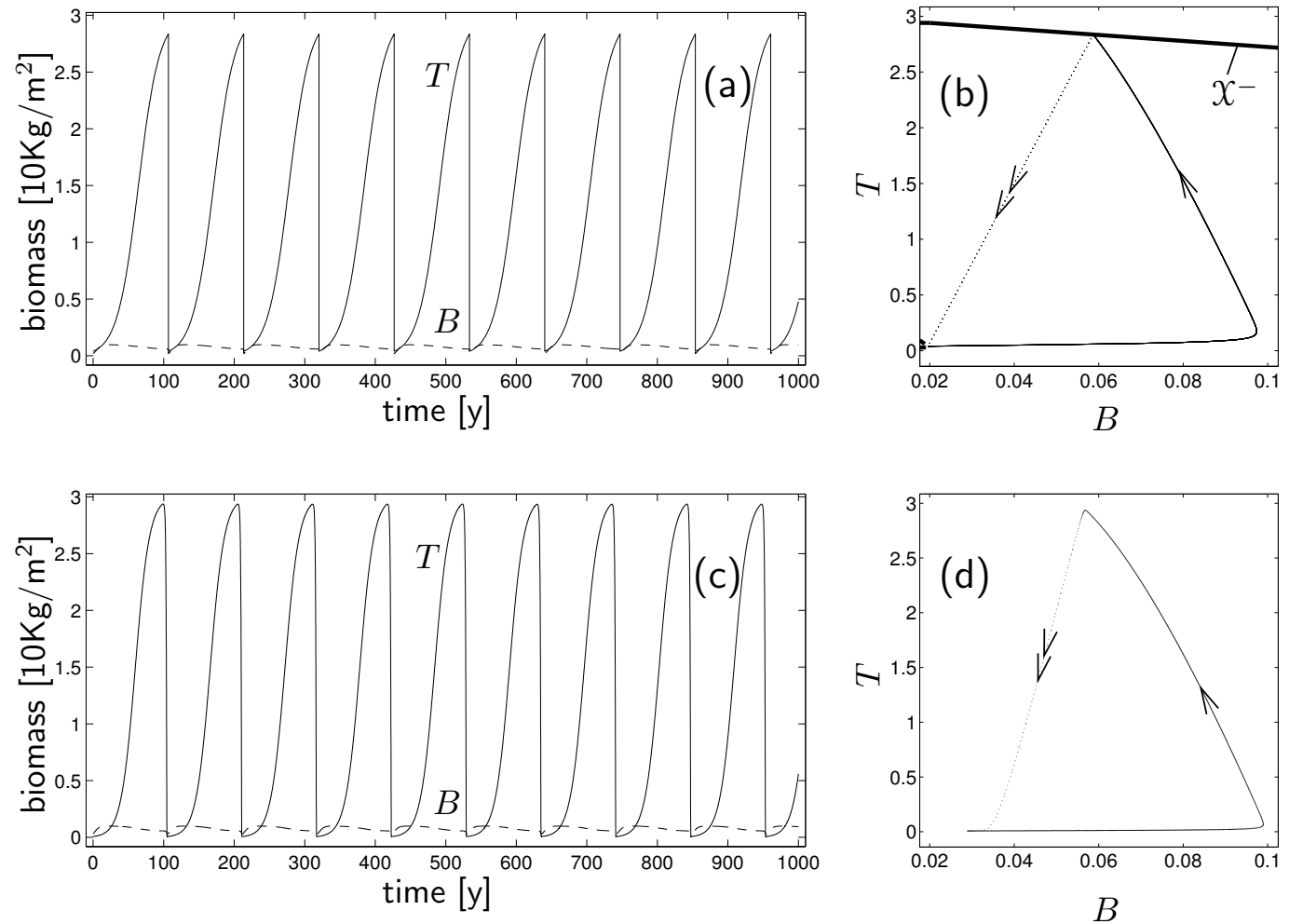

Figure 3. Maggi \& Rinaldi 2005 

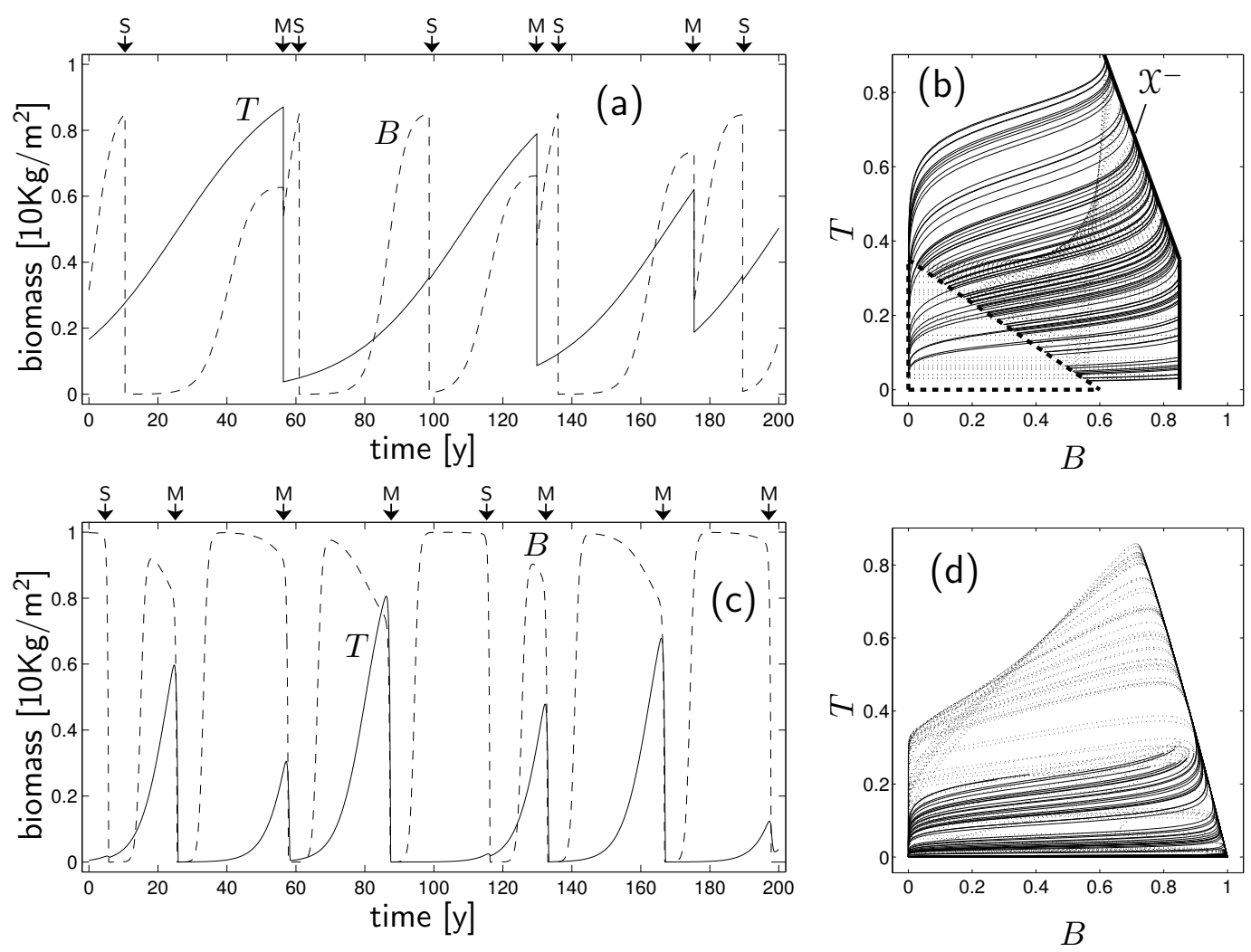

Figure 4. Maggi \& Rinaldi 2005 

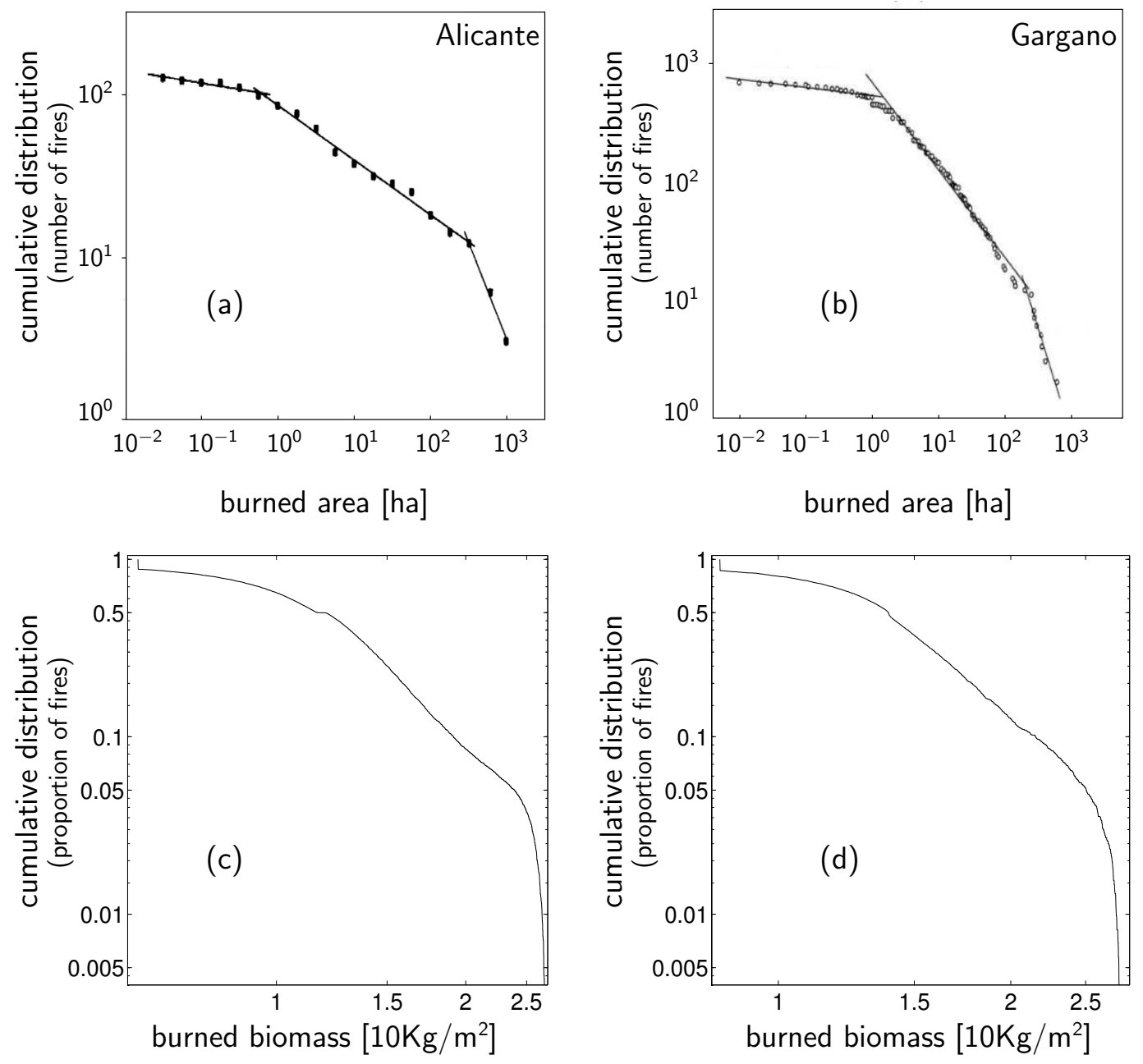

Figure 5. Maggi \& Rinaldi 2005 


\section{Appendix}

The fourth-order model described in Casagrandi and Rinaldi (1999) is the following

$$
\begin{aligned}
\dot{B} & =r_{B} B\left(1-\frac{B}{K_{B}}\right)-\alpha B T-\beta_{B} \frac{B}{B+h_{B B}} F_{B}-\gamma_{B} \frac{B}{B+h_{B T}} F_{T} \\
\dot{T} & =r_{T} T\left(1-\frac{T}{K_{T}}\right)-\beta_{T} \frac{T}{T+h_{T T}} F_{T}-\gamma_{T} \frac{T}{T+h_{T B}} F_{B} \\
\dot{F}_{B} & =\beta_{B} \frac{B}{B+h_{B B}} F_{B}+\gamma_{B} \frac{B}{B+h_{B T}} F_{T}-\delta_{B} F_{B} \\
\dot{F}_{T} & =\beta_{T} \frac{T}{T+h_{T T}} F_{T}+\gamma_{T} \frac{T}{T+h_{T B}} F_{B}-\delta_{T} F_{T}
\end{aligned}
$$

where $B$ and $T\left[F_{B}\right.$ and $\left.F_{T}\right]$ are the non-burning [burning] biomasses of the lower and upper layers of the forest, and all lower-case letters, as well as $K_{B}$ and $K_{T}$, are constant parameters. If there is no fire, i.e. if $F_{B}=F_{T}=0$, the model describes the growing phase of the forest and reduces to eqs. (3).

If we are interested in determining the pre-fire manifold $\mathcal{X}^{-}$, we must simply determine the pairs $(B, T)$ at which a growing forest settles the conditions for fire ignition and propagation. For this we can analyze eqs. (A.3, A.4) which describe the dynamics of the fire. If there is no fire, system (A.3, A.4) with $B$ and $T$ frozen at their current values, is at the trivial equilibrium $F_{B}=F_{T}=0$. If this trivial equilibrium is stable, a small accidental fire (i.e. a small positive perturbation of $F_{B}$ and $F_{T}$ ) will not have the chance to propagate. By contrast, the same small accidental fire will propagate if the trivial equilibrium is unstable. Thus, the pre-fire manifold $X^{-}$can be interpreted as the set of values $(B, T)$ at which the trivial equilibrium of system (A.3, A.4) becomes unstable. But system (A.3, A.4) with $B$ and $T$ 
frozen is a linear system with Jacobian matrix given by

$$
J=\left[\begin{array}{cc}
\beta_{B} \frac{B}{B+h_{B B}}-\delta_{B} & \gamma_{B} \frac{B}{B+h_{B T}} \\
\\
\gamma_{T} \frac{T}{T+h_{T B}} & \beta_{T} \frac{T}{T+h_{T T}}-\delta_{T}
\end{array}\right]
$$

For relatively small values of $B$ and $T$, the diagonal elements of $J$ are negative (see Casagrandi and Rinaldi (1999)) since $\delta_{B}$ and $\delta_{T}$ are high. Indeed, $\delta_{B}$ and $\delta_{T}$ are the rates of decay of the fires when there is nothing to burn (i.e. $\dot{F}_{B}=-\delta_{B} F_{B}$ if $B=T=0$, and similarly for crown fires). Thus, the transition to instability of the Jacobian matrix is simply revealed by the annihilation of its determinant, i.e.

$$
\left(\beta_{B} \frac{B}{B+h_{B B}}-\delta_{B}\right)\left(\beta_{T} \frac{T}{T+h_{T T}}-\delta_{T}\right)-\gamma_{B} \gamma_{T} \frac{B}{B+h_{B T}} \frac{T}{T+h_{T B}}=0
$$

This condition implicitly defines a function $T(B)$ which corresponds to the pre-fire manifold $X^{-}$. Taking the first and second derivatives with respect to $B$ of eq. (A.5) with $T=T(B)$, one obtains two relationships involving $T^{\prime}(B)$ and $T^{\prime \prime}(B)$, which can be used to prove that

$$
T^{\prime}(B)<0 \quad T^{\prime \prime}(B)<0
$$

i.e. that the function $T(B)$ is decreasing and the set $T \leq T(B)$ is convex. The proof is quite simple if $h_{B B}=h_{T T}=h_{B T}=h_{T B}=h$, which is actually the case considered in all simulations of Mediterranean forests presented in Casagrandi and Rinaldi (1999). In fact, in such a case, eq. (A.5) can be solved with respect to $T$ and gives

$$
T=\frac{a B-b}{c B-d}
$$


where

$$
\begin{aligned}
& a=\left(\delta_{T}+h\right)\left(\beta_{B}-\delta_{B}\right) \\
& b=\left(\delta_{T}+h\right) \delta_{B} h \\
& c=\left(\beta_{T}-\delta_{T}\right)\left(\beta_{B}-\delta_{B}\right)-\gamma_{B} \gamma_{T} \\
& d=\left(\beta_{T}-\delta_{T}\right)
\end{aligned}
$$

Assuming that each layer can burn provided its biomass is infinitely large, from eqs. (A.3) and (A.4) we obtain

$$
\beta_{B}>\delta_{B} \quad \beta_{T}>\delta_{T}
$$

which imply that the 4 parameters $a, b, c, d$ are positive, since $\gamma_{B}$ and $\gamma_{T}$ (the inter-layers fire attack rates) are small (Casagrandi and Rinaldi (1999)). Moreover,

$$
\frac{b c}{a d}=\delta_{B} h-\frac{\gamma_{B} \gamma_{T}}{\left(\beta_{B}-\delta_{B}\right)\left(\beta_{T}-\delta_{T}\right)}<\delta_{B} h
$$

so that

$$
b c<a d
$$

since $\delta_{B} h$ is smaller than 1 (see Table 2 in Casagrandi and Rinaldi (1999)). Thus, from eq. (A.6) it follows that the function $T(B)$ is positive in the interval

$$
0<B<\frac{b}{a}
$$

while its first and second derivatives

$$
T^{\prime}(B)=\frac{b c-a d}{(c B-d)^{2}} \quad T^{\prime \prime}(B)=\frac{2(a d-b c) c}{(c B-d)^{3}}
$$

are negative in the same interval.

In conclusion, the fourth-order model of Casagrandi and Rinaldi (1999) suggests that the pre-fire manifold $X^{-}$is a smooth manifold decreasing with 
respect to $B$ and that the set below the manifold $X^{-}$is a convex set. 